\title{
Robotic-assisted interval cytoreductive surgery in ovarian cancer: a feasibility study
}

Semiramis L. Carbajal-Mamani, MD¹, David Schweer, MD², Merry J. Markham, MD , Ashwini K. Esnakula, MD³, Joseph R. Grajo, MD${ }^{4}$, Jacqueline C. Castagno, MD², Joel Cardenas-Goicoechea, MD²

${ }^{1}$ Department of Medicine, ${ }^{2}$ Division of Gynecologic Oncology, Department of Obstetrics and Gynecology, ${ }^{3}$ Department of Pathology, Immunology and Laboratory Medicine, ${ }^{4}$ Department of Radiology, College of Medicine, University of Florida, Gainesville, FL, USA

\section{Objective}

The primary objective was to assess the feasibility of robotic-assisted interval cytoreductive surgery for achieving complete cytoreduction for patients with advanced-stage ovarian cancer. The secondary objective was to examine the perioperative outcomes.

Methods

A retrospective study of 12 patients with stage IIIC or IV ovarian, fallopian tube, and primary peritoneal carcinoma who underwent interval cytoreductive surgery after neo-adjuvant chemotherapy.

Results

Optimal cytoreduction was achieved in $100 \%$ of selected patients. Complete cytoreductive surgery was achieved in $75 \%$ of patients. The estimated mean blood loss was $100 \mathrm{~mL}$. The median length of hospital stay was 2 days. Perioperative complication and 30-day readmission rates were $8.3 \%$ (1 patient). The median follow-up time was 9.5 months.

\section{Conclusion}

Robotic-assisted interval cytoreductive surgery in ovarian cancer is safe and feasible and may be an alternative to standard laparotomy in selected patients.

Keywords: Robotic surgical procedure; Ovarian cancer; Cytoreductive surgical procedure

\section{Introduction}

Ovarian cancer is the most lethal form of gynecologic cancer. In 2019, the American Cancer Society estimated 22,530 new ovarian cancer cases and 13,980 ovarian cancer deaths [1]. The management of ovarian cancer has been evolving with the introduction of new drugs and surgical innovation.

Patients with advanced-stage ovarian cancer traditionally underwent surgery upfront with maximal cytoreductive surgery via laparotomy (open surgery) followed by adjuvant platinum-based chemotherapy. A recently emerging approach involves neo-adjuvant chemotherapy followed by interval tumor debulking. Phase III clinical trials showed similar progression-free and overall survival rates for this emerging modality, but surgery following neo-adjuvant chemotherapy features lower morbidity and mortality rates than the tradi- tional method [2-5]. Current guidelines endorse neo-adjuvant chemotherapy as an option for some patients [6]. The ultimate goal is to achieve complete cytoreductive surgery,

Received: 2019.05.29. Revised: 2019.11.01. Accepted: 2019.12.11. Corresponding author: Joel Cardenas-Goicoechea, MD

Division of Gynecologic Oncology, Department of Obstetrics and Gynecology, College of Medicine, University of Florida, 1600 SW Archer Rd m509, Gainesville, FL 32610, USA

E-mail: joelcardenas@ufl.edu

https://orcid.org/0000-0002-0589-5785

Articles published in Obstet Gynecol Sci are open-access, distributed under the terms of the Creative Commons Attribution Non-Commercial License (http://creativecommons. org/licenses/by-nc/3.0/) which permits unrestricted non-commercial use, distribution, and reproduction in any medium, provided the original work is properly cited.

Copyright $\odot 2020$ Korean Society of Obstetrics and Gynecology 


\section{Obstetrics \& Gynecology Science}

Semiramis L. Carbajal-Mamani, et al. Robotic-assisted cytoreduction in ovarian cancer

which has been shown to improve survival [7-9].

The morbidity and surgical complexity associated with interval cytoreductive surgery appears lower than that with surgery upfront $[2,4,10]$. As a result, patients and gynecologic oncologists may be inclined to use the minimally invasive approach to achieve similar oncologic outcomes as laparotomy. Furthermore, patients with significant comorbidities and elderly patients may decline to undergo laparotomy. The benefits of minimally invasive surgery over laparotomy are reduced blood loss, reduced post-operative pain, a smaller incision with a reduced risk of wound infection, a shorter hospital stay, a faster recovery, and a shorter time to resuming chemotherapy after interval cytoreductive surgery.

Phase III trials that compare standard laparotomy with minimally invasive surgery for ovarian cancer after neo-adjuvant chemotherapy are lacking. Oncologic outcomes have been reported from the National Cancer Database [11]. A phase II clinical trial by Gueli Alletti et al. [12] of 30 patients demonstrated that minimally invasive surgery is safe and feasible in ovarian cancer patients with complete clinical response to neo-adjuvant chemotherapy. Furthermore, the latest version of the National Comprehensive Cancer Network's guidelines for ovarian cancer considers that there may be a role for minimally invasive surgery in ovarian cancer. Patients who are unable to be optimally debulked using minimally invasive techniques should be converted to an open procedure performed by an experienced surgeon [13].

Surgical innovation is important, as are outcomes, but complex $[14,15]$. Robotic-assisted surgery has technological differences with laparoscopy, such as 3-dimensional optics, improved depth perception, camera stability, increased dexterity, and tremor filtration. Furthermore, the latest robotic platform Xi (Intuitive Surgical, Inc., Sunnyvale, CA, USA) allows pelvic and upper abdominal surgery with simple maneuvers. Very limited data are available with this new platform in ovarian cancer. This study aimed to assess complete cytoreductive surgery and perioperative outcomes with the robotic platform Xi in advanced-stage ovarian cancer patients after neo-adjuvant chemotherapy.

\section{Materials and methods}

\section{Objective}

The primary objective of the study was to assess the feasi- bility of robotic-assisted interval cytoreductive surgery for patients with advanced ovarian cancer after neo-adjuvant chemotherapy. The secondary objective was to examine perioperative outcomes.

\section{Eligibility criteria}

Patients with biopsy-proven stage IIIC or IV ovarian, fallopian tube, and primary peritoneal carcinoma with a clinical response to neo-adjuvant chemotherapy who would also undergo interval cytoreductive surgery with robotic assistance were included in this study. The minimum follow-up duration after surgery was 60 days. Patients with clinical early-stage cancer (I to IIIB), primary debulking surgery, and secondary cytoreductive surgery were excluded. Patients with resolution of ascites, pleural effusion, significant decrease of cancer antigen 125 (CA-125), decrease of tumor burden on computed tomography (CT) or a positron emission tomography/ CT scan, and partial or complete response to chemotherapy by response evaluation criteria in solid tumors (RECIST) criteria after neo-adjuvant chemotherapy underwent diagnostic laparoscopy. Patients underwent robotic-assisted surgery if the diagnostic laparoscopy showed potential complete cytoreductive surgery. Study criteria were based on those of the randomized controlled trial by Rutten et al. [16]. All eligible patients identified during the study period were included.

\section{Data extraction}

Data were abstracted from the patients' electronic medical records in Epic (Epic Systems Corporation, Verona, WI, USA). The variables were: age, comorbid conditions (hypertension, diabetes, chronic obstructive pulmonary disease, asthma, and coronary artery disease), prior abdominopelvic surgery, body mass index (BMI), American Society of Anesthesiologists (ASA) score, RECIST tumor response, CA-125 at the time of diagnosis and pre-operatively, chemotherapy, breast cancer gene status, tumor histology and grade, operative time, blood loss, transfusion, length of hospital stay, complication, readmission, surgical procedure, complexity score, and pathology. Chemotherapy response score was assessed by a pathologist (AE) during the review of a single omental tissue block with the least response to chemotherapy as per the recent recommended guidelines [17].

The surgical approach for robotic-assisted surgery followed the standard approach described in the National Comprehensive Cancer Network's 2019 guidelines [13]. Pre- and post- 


\title{
Obstetrics \& Gynecology Science
}

\author{
Vol. 63 , No. 2, 2020
}

operative care were performed according to enhanced recovery after surgery protocols $[18,19]$. To define case complexity, we used the surgical complexity scoring system described by Aletti et al. [20]. The da Vinci Xi robotic platform was used. Port placement was described by Lucidi et al. [21].

Complete cytoreductive surgery was defined as no gross residual disease at the end of surgery, optimal cytoreductive surgery was defined as no residual disease measuring greater than $1 \mathrm{~cm}$, and sub-optimal cytoreductive surgery was defined as residual disease measuring greater than $1 \mathrm{~cm}$.

\section{Data analysis}

Categorical variables are presented as number of cases and percentages. Continuous variables are presented as median, mean, standard deviation, range, and 95\% confidence interval $(\mathrm{CI})$. For categorical variables, the $\chi^{2}$ test and Fisher's exact test were used. The data analysis was performed using SPSS Statistics version 25 (SPSS Inc., Chicago, IL, USA).

\section{Results}

During the study period, 12 patients met the inclusion criteria and were included in the study. The median follow-up was 9.5 months $(95 \% \mathrm{Cl}, 6.19-13.31)$. Three patients in the robotic group underwent diagnostic laparoscopy upon receiving the initial diagnosis. The criteria for neo-adjuvant chemotherapy included a low likelihood of optimal cytoreduction on diagnostic laparoscopy (3/12), stage IV disease (3/12), tumor in porta hepatis on a CT scan (1/12), unresectable tumor upon exploratory laparotomy (1/12), acute venous thromboembolism upon diagnosis (2/12), and patient declining surgery up front (1/12) (Supplementary Table 1).

Patient demographics are presented in Table 1. Median age was 72.5 years, median BMI was $23.35 \mathrm{~kg} / \mathrm{m}^{2}$ (17.53-41.28), and all patients had an ASA score of 3. Perioperative outcomes are presented in Table 2. The estimated mean blood loss was $100 \mathrm{~mL}$ (50-800 mL). Pre- and post-operative changes in hemoglobin were $1.5 \pm 0.5(95 \% \mathrm{Cl}, 1-1.7)$. Patients spent a median 2 days in the hospital. The 30 -day post-operative complication and readmission rate was $8.3 \%$ (1 patient). Complexity scores of surgical procedures performed is in Supplementary Table 2.

One patient required a post-operative blood transfusion. One patient was readmitted with bacteremia. Another pa- tient required surgery due to port site hernia on post-operative day 31. The patient was admitted and underwent lapa-

Table 1. Patient demographics

\begin{tabular}{|c|c|}
\hline Variables & Robotic $(n=12)$ \\
\hline Age & $72.5(54-85)$ \\
\hline BMI $\left(\mathrm{kg} / \mathrm{m}^{2}\right)$ & $23.35(17.53-41.28)$ \\
\hline \multicolumn{2}{|l|}{ Medical co-morbid condition } \\
\hline Yes & $10(83.3)$ \\
\hline \multicolumn{2}{|l|}{ Previous abdominal/pelvic surgery } \\
\hline Yes & $9(75)$ \\
\hline \multicolumn{2}{|l|}{ ASA score } \\
\hline 2 & 0 \\
\hline 3 & $12(100)$ \\
\hline 4 & 0 \\
\hline CA-125 at diagnosis (UI/mL) & $931(264-6,987)$ \\
\hline Pre-operative CA-125 (Ul/mL) & $14(5-240)$ \\
\hline \multicolumn{2}{|l|}{ RECIST tumor response } \\
\hline Complete & $1(8.3)$ \\
\hline Partial & $9(75)$ \\
\hline Stable & $1(8.3)$ \\
\hline No. of cycles of NACT & $3.5(3-6)$ \\
\hline No. of cycles of adjuvant chemotherapy & $2.5(0-4)$ \\
\hline \multicolumn{2}{|l|}{ Type of chemotherapy } \\
\hline Carboplatin-paclitaxel every 3 weeks & $11(91.7)$ \\
\hline Carboplatin-paclitaxel every week & $1(8.3)$ \\
\hline $\begin{array}{l}\text { Carboplatin-paclitaxel-bevacizumab every } \\
3 \text { weeks }\end{array}$ & 0 \\
\hline \multicolumn{2}{|l|}{ BRCA status } \\
\hline No pathogenic variant & $9(75)$ \\
\hline BRCA1 & $2(17)$ \\
\hline BRCA2 & $0(0)$ \\
\hline Unknown & $1(8)$ \\
\hline \multicolumn{2}{|l|}{ Histology } \\
\hline Serous & $12(100)$ \\
\hline Clear cell & 0 \\
\hline Carcinosarcoma & 0 \\
\hline Mucinous & 0 \\
\hline \multicolumn{2}{|l|}{ High grade } \\
\hline Yes & $12(100)$ \\
\hline
\end{tabular}

Values are presented as median (range) or number (\%). BMI, body mass index; ASA, American Society of Anesthesiologists; NACT, neoadjuvant chemotherapy; CA-125, cancer antigen 125; RECIST, response evaluation criteria in solid tumors. 


\section{Obstetrics \& Gynecology Science}

Semiramis L. Carbajal-Mamani, et al. Robotic-assisted cytoreduction in ovarian cancer

roscopic surgery at an outside hospital. The port site hernia was induced by the 12-mm assistant port. As a result of that event, institutional practice changed to close the assistant port with 0-vicryl suture. It is possible that the port size was extended due to the abdominopelvic surgery.

Optimal cytoreductive surgery was achieved in 100\% of patients; including optimal but not complete cytoreduction in $3(25 \%)$ (Table 3). Complete cytoreduction was achieved in 9 cases (75\%), with no gross residual disease. Two robotic cases required supra-umbilical laparotomy to achieve optimal cytoreduction. In both cases, the pelvic surgery was performed using the Xi robotic platform. One case required a transverse colon resection and partial gastrectomy, while the other required small bowel resection. The other 3 cases did not require laparotomy; rather, one each required partial transverse colon resection, partial cecal resection with appendectomy, and appendectomy (Supplementary Table 3).

The abstracted data did not provide a standardized and objective confirmation of complete resection. However, all patients underwent a CT scan at 2-20 weeks post-operative (median, 8 weeks; 95\% Cl, 5.61-12.06). All cases show no measurable disease. One case showed ascites. All patients underwent CA-125 testing at 4 weeks post-operative. Eleven of 12 patients had normal levels. One patient had a pre-

Table 2. Perioperative outcomes

\begin{tabular}{|c|c|}
\hline Outcomes & Robotic $(n=12)$ \\
\hline Operative time (min) & $237.5(128-446)$ \\
\hline Estimated blood loss (mL) & $100(50-800)$ \\
\hline $\begin{array}{l}\text { Change in hemoglobin pre-op and } \\
\text { post-op ( } \mathrm{g} / \mathrm{dL})\end{array}$ & $1.55(0.6-2.3)$ \\
\hline \multicolumn{2}{|l|}{ Blood transfusion } \\
\hline Yes & $2(16.7)$ \\
\hline Length of hospital stay (days) & $2(1-7)$ \\
\hline \multicolumn{2}{|l|}{ Hospital complication } \\
\hline Yes & $1(8.3)$ \\
\hline \multicolumn{2}{|l|}{ 30-day post-op complication } \\
\hline Yes & $1(8.3)$ \\
\hline \multicolumn{2}{|l|}{ 30-day readmission } \\
\hline Yes & $1(8.3)$ \\
\hline \multicolumn{2}{|l|}{ Follow-up } \\
\hline Median (mon) & $9.5(2-17)$ \\
\hline Mean \pm standard deviation & $9.75 \pm 5.61$ \\
\hline
\end{tabular}

Values are presented as median (range) or number (\%). operative CA-125 level of $240 \mathrm{U} / \mathrm{mL}$ and a 4 weeks postoperative level of $84 \mathrm{U} / \mathrm{mL}$ (Supplementary Table 3). Patients received a median of 2.5 cycles of adjuvant platinum-based chemotherapy $(95 \% \mathrm{Cl}, 0.89-2.95$; range, $0-4)$. One patient declined adjuvant chemotherapy. Two patients received 6 cycles of neo-adjuvant chemotherapy and 0 after surgery (Supplementary Table 2).

The pathologic findings showed that $83 \%$ of patients had a positive response, with chemotherapy response scores of 2 and 3 (Table 4). With a median follow-up of 9.5 months, $92 \%$ of the patients remained alive (Table 5 ). Three patients underwent lymph node dissection due to abnormal findings during surgery or CT scan findings (Supplementary Table 3). One case had a 2-mm metastatic focus of carcinoma in a left pelvic lymph node. In another case, 2 pelvic nodes failed to reveal any carcinoma. In another case, 5 lymph nodes were removed; 1 of them included a $0.35-\mathrm{mm}$ metastatic serous carcinoma. The most common location of disease recurrence was the peritoneum (4/12), followed by lymph nodes in the

Table 3. Surgical outcomes

\begin{tabular}{|c|c|}
\hline Outcomes & Robotic $(n=12)$ \\
\hline \multicolumn{2}{|l|}{ Surgical procedure } \\
\hline Hysterectomy, salpingo-oophorectomy & 0 \\
\hline $\begin{array}{l}\text { Hysterectomy, salpingo-oophorectomy, nodes, } \\
\text { omentum }\end{array}$ & $5(41.7)$ \\
\hline $\begin{array}{l}\text { Hysterectomy, salpingo-oophorectomy, } \\
\text { omentum }\end{array}$ & $3(25)$ \\
\hline Oophorectomy, omentum & $4(33.3)$ \\
\hline Oophorectomy, nodes, omentum & 0 \\
\hline \multicolumn{2}{|l|}{ Bowel surgery } \\
\hline Yes & $5(41.7)$ \\
\hline Intra-operative complication & 0 \\
\hline \multicolumn{2}{|l|}{ Conversion $^{\mathrm{a})}$} \\
\hline Yes & $2(16.7)$ \\
\hline \multicolumn{2}{|l|}{ Cytoreduction at the completion of surgery } \\
\hline Complete & $9(75)$ \\
\hline Optimal & $3(25)$ \\
\hline Suboptimal & 0 \\
\hline \multicolumn{2}{|l|}{ Complexity score } \\
\hline 1 (low) & $7(58.3)$ \\
\hline 2 (intermediate) & $5(41.7)$ \\
\hline
\end{tabular}




\section{Obstetrics \& Gynecology Science}

Vol. 63, No. 2, 2020

abdomen and pelvis (1/12) and chest (1/12) (Supplementary Table 3).

\section{Discussion}

The surgical approach for interval cytoreduction in ovarian cancer patients is evolving. Our preliminary data showed that complete cytoreductive surgery is feasible $(75 \%)$ and may be an alternative approach for some patients.

Robotic-assisted surgery for ovarian cancer was previously described but without a focus on interval cytoreductive surgery [22-24]. Our data are among the first to address the role of robotic surgery using the da Vinci Xi platform and overcomes the limitations of previous publications based on an older platform. We believe this is relevant since the neoadjuvant chemotherapy rate has increased.

Table 4. Histological findings from Interval cytoreductive surgery

\begin{tabular}{ll}
\hline Outcomes & Robotic $(\mathbf{n}=\mathbf{1 2})$ \\
\hline Chemotherapy response score & $2(16.7)$ \\
1 & $7(58.3)$ \\
2 & $3(25)$ \\
3 & \\
Residual disease & $10(83.3)$ \\
Macroscopic & $2(16.7)$ \\
Microscopic & \\
Pathologic stage & 0 \\
0 & $2(16.7)$ \\
IA & $1(8.3)$ \\
IIC & 0 \\
IIIA & $1(8.3)$ \\
IIIB & $7(58.3)$ \\
IIIC & $1(8.3)$ \\
IV &
\end{tabular}

Values are presented as number (\%).

Table 5. Survival data

\begin{tabular}{lc}
\hline Status at last follow-up & Robotic $(\mathbf{n = 1 2 )}$ \\
\hline No evidence of disease & $6(50)$ \\
Alive with disease & $5(41.7)$ \\
Deceased & $1(8.3)$ \\
\hline
\end{tabular}

Values are presented as number (\%).
The robotic approach may be an additional tool for achieving complete cytoreduction. Our preliminary data are consistent with published data that included mainly laparoscopic cytoreductive surgery $(74.50 \% ; 95 \% \mathrm{Cl}, 40.41-97.65)$ $[11,12,25-29]$. Although phase III trials demonstrated that interval cytoreductive surgery is less invasive and required fewer surgeries than debulking upfront, adequate exposure, identification of tumors in the upper abdomen, and removal of residual disease are critical $[2,4]$.

The feasibility of performing complex and widespread surgery, such as bowel and stomach resection, diaphragm stripping, splenectomy, and pelvic and para-aortic node dissection, may be limited for minimally invasive surgery. Our 2 robotic cases that required supra-umbilical laparotomy were due to the presence of a tumor in the upper abdomen that required bowel resection, while 1 case required transverse colon and partial stomach resection. Brown et al. [30] reported a 17\% conversion rate to achieve optimal cytoreduction among 53 patients who underwent laparoscopic surgery. Ackroyd et al. [25] reported a 3\% conversion rate (3 of 29 cases) and the need for a mini-laparotomy in 17\% (5 of 29 cases) of patients who underwent robotic interval cytoreduction.

Our 30-day perioperative outcomes are similar to published data. Our readmission rate $(8.3 \%)$ is within the range of published data (5-14\%) [11,25].

Overall, the reported hospital stay was 2-4 days for minimally invasive surgery $[11,12,25-28,30]$. Our overall hospital stay was 2 days (median, 2; mean, 2.5; $95 \% \mathrm{Cl}, 1.27-3.73$ ). These findings may be due to the implementation of enhanced recovery after surgery guidelines and represent a relatively shorter stay than other experiences of 5-6 days $[10,11,24,27,30]$. In the largest study, which included 2,621 patients in the laparotomy group versus 450 cases in the laparoscopy group, the reported length of hospital stay was 5 days (range, 4-7 days) and 4 days (range, 2-6 days) for laparotomy and laparoscopy, respectively $(P<0.001)$ [11].

Elderly patients with significant comorbidities, patients older than 80 years, and frail patients may decline open surgery, so minimally invasive surgery may be an option. In our study, patients in the robotic group tended to be older and have higher ASA scores than those in previous publications (Table 1) [12].

A recent international study from 5 centers collected data regarding 123 patients over 8 years of age with a minimum 


\section{Obstetrics \& Gynecology Science}

Semiramis L. Carbajal-Mamani, et al. Robotic-assisted cytoreduction in ovarian cancer

of 6 months of follow-up (3 cases per center per year). Investigators assessed the feasibility and outcomes of minimally invasive surgery after neo-adjuvant chemotherapy. A total of 122 patients (96.1\%) had no residual tumors after surgery. With a median follow-up of 37 months (7-86 months), the median progression-free survival was 23 months and 5 -year survival was $52 \%$ [31].

The limitations of our preliminary experience are inherent to its retrospective design and small case cohort. However, limited data exist for assessing the robotic platform in this setting $[22,23]$. Furthermore, given the introduction of the da Vinci Xi system and widespread adoption of robotic surgery, more data are expected. We continue to collect data to assess disease recurrence and survival.

The strengths of this study include its enrollment of consecutive cases performed by the same surgical team using a standardized approach, which produced consistent, precise, and reliable data. Likewise, our focus on using the da Vinci Xi platform, which minimizes maneuvers for upper abdominal surgery, is another asset of this study.

Our preliminary data suggest that robotic-assisted surgery is an option to achieve complete interval cytoreductive surgery with similar perioperative outcomes to those of laparotomy, the gold standard approach. Prospective trials with adequate follow-up durations are needed to confirm our results as well as assess tumor recurrence and survival.

\section{Acknowledgments}

The authors thank Bernie Amaro, Research Administrative Assistant, University of Florida Department of Obstetrics and Gynecology, for his technical and editorial assistance preparing this paper for publication.

\section{Conflict of Interest}

No potential conflict of interest relevant to this article was reported.

\section{Ethical approval}

The University of Florida Institutional Review Board approved this study (\#IRB201702519). The study period lasted 2 years (2017-2018).

\section{Patient consent}

This retrospective study was preceded by a written protocol that was approved.

\section{Supplementary materials}

Supplementary Tables associated with this article can be found online at https://doi.org/10.5468/ogs.2020.63.2.150.

\section{References}

1. Siegel RL, Miller KD, Jemal A. Cancer statistics, 2019. CA Cancer J Clin 2019;69:7-34.

2. Vergote I, Tropé CG, Amant F, Kristensen GB, Ehlen T, Johnson $\mathrm{N}$, et al. Neoadjuvant chemotherapy or primary surgery in stage IIIC or IV ovarian cancer. N Engl J Med 2010;363:943-53.

3. Kehoe S, Hook J, Nankivell M, Jayson GC, Kitchener $H$, Lopes $T$, et al. Primary chemotherapy versus primary surgery for newly diagnosed advanced ovarian cancer (CHORUS): an open-label, randomised, controlled, noninferiority trial. Lancet 2015;386:249-57.

4. Onda T, Satoh T, Saito T, Kasamatsu T, Nakanishi T, Nakamura $\mathrm{K}$, et al. Comparison of treatment invasiveness between upfront debulking surgery versus interval debulking surgery following neoadjuvant chemotherapy for stage III/IV ovarian, tubal, and peritoneal cancers in a phase III randomised trial: Japan Clinical Oncology Group Study JCOG0602. Eur J Cancer 2016;64:22-31.

5. Vergote I, Coens C, Nankivell M, Kristensen GB, Parmar MK, Ehlen $T$, et al. Neoadjuvant chemotherapy versus debulking surgery in advanced tubo-ovarian cancers: pooled analysis of individual patient data from the EORTC 55971 and CHORUS trials. Lancet Oncol 2018;19:1680-7.

6. Wright AA, Bohlke K, Armstrong DK, Bookman MA, Cliby WA, Coleman RL, et al. Neoadjuvant chemotherapy for newly diagnosed, advanced ovarian cancer: Society 


\section{Obstetrics \& Gynecology Science}

Vol. 63, No. 2, 2020

of Gynecologic Oncology and American Society of Clinical Oncology clinical practice guideline. J Clin Oncol 2016;34:3460-73.

7. Chang SJ, Bristow RE. Evolution of surgical treatment paradigms for advanced-stage ovarian cancer: redefining 'optimal' residual disease. Gynecol Oncol 2012;125:483-92.

8. Hamilton CA, Miller A, Casablanca Y, Horowitz NS, Rungruang $B$, Krivak TC, et al. Clinicopathologic characteristics associated with long-term survival in advanced epithelial ovarian cancer: an NRG Oncology/Gynecologic Oncology Group ancillary data study. Gynecol Oncol 2018;148:275-80.

9. Schorge JO, Bregar AJ, Durfee J, Berkowitz RS. Meigs to modern times: the evolution of debulking surgery in advanced ovarian cancer. Gynecol Oncol 2018;149:447-54.

10. Fagotti A, Ferrandina G, Vizzielli G, Fanfani F, Gallotta V, Chiantera V, et al. Phase III randomised clinical trial comparing primary surgery versus neoadjuvant chemotherapy in advanced epithelial ovarian cancer with high tumour load (SCORPION trial): final analysis of perioperative outcome. Eur J Cancer 2016;59:22-33.

11. Melamed A, Nitecki R, Boruta DM 2nd, Del Carmen MG, Clark RM, Growdon WB, et al. Laparoscopy compared with laparotomy for debulking ovarian cancer after neoadjuvant chemotherapy. Obstet Gynecol 2017;129:861-9.

12. Gueli Alletti S, Bottoni C, Fanfani F, Gallotta V, Chiantera $\mathrm{V}$, Costantini $\mathrm{B}$, et al. Minimally invasive interval debulking surgery in ovarian neoplasm (MISSION trialNCT02324595): a feasibility study. Am J Obstet Gynecol 2016;214:503.e1-503.e6.

13. National Comprehensive Cancer Network. National Comprehensive Cancer Network's practice guidelines in oncology. Ovarian cancer [Internet]. Plymouth Meeting (PA): National Comprehensive Cancer Network; c2018 [cited 2019 May 1]. Available from: https://www.nccn. org/professionals/physician_gls/pdf/ovarian.pdf.

14. Ergina PL, Cook JA, Blazeby JM, Boutron I, Clavien PA, Reeves $B C$, et al. Challenges in evaluating surgical innovation. Lancet 2009;374:1097-104.

15. Garas G, Cingolani I, Panzarasa P, Darzi A, Athanasiou T. Network analysis of surgical innovation: measuring value and the virality of diffusion in robotic surgery. PLoS One 2017;12:e0183332.

16. Rutten MJ, van Meurs HS, van de Vrie R, Gaarenstroom KN, Naaktgeboren CA, van Gorp T, et al. Laparoscopy to predict the result of primary cytoreductive surgery in patients with advanced ovarian cancer: a randomized controlled trial. J Clin Oncol 2017;35:613-21.

17. Böhm S, Faruqi A, Said I, Lockley M, Brockbank E, Jeyarajah $A$, et al. Chemotherapy response score: development and validation of a system to quantify histopathologic response to neoadjuvant chemotherapy in tubo-ovarian high-grade serous carcinoma. J Clin Oncol 2015;33:2457-63.

18. Nelson G, Altman AD, Nick A, Meyer LA, Ramirez PT, Achtari $C$, et al. Guidelines for pre- and intra-operative care in gynecologic/oncology surgery: Enhanced Recovery After Surgery (ERAS () ) Society recommendations-part I. Gynecol Oncol 2016;140:313-22.

19. Nelson G, Altman AD, Nick A, Meyer LA, Ramirez PT, Achtari $C$, et al. Guidelines for postoperative care in gynecologic/oncology surgery: Enhanced Recovery After Surgery (ERAS $\AA$ ) Society recommendations--part II. Gynecol Oncol 2016;140:323-32.

20. Aletti GD, Santillan A, Eisenhauer EL, Hu J, Aletti G, Podratz KC, et al. A new frontier for quality of care in gynecologic oncology surgery: multi-institutional assessment of short-term outcomes for ovarian cancer using a risk-adjusted model. Gynecol Oncol 2007;107:99-106.

21. Lucidi A, Chiantera V, Gallotta V, Ercoli A, Scambia G, Fagotti $A$. Role of robotic surgery in ovarian malignancy. Best Pract Res Clin Obstet Gynaecol 2017;45:74-82.

22. Magrina JF, Zanagnolo V, Noble BN, Kho RM, Magtibay P. Robotic approach for ovarian cancer: perioperative and survival results and comparison with laparoscopy and laparotomy. Gynecol Oncol 2011;121:100-5.

23. Nezhat FR, Finger TN, Vetere $P$, Radjabi $A R$, Vega $M$, Averbuch $L$, et al. Comparison of perioperative outcomes and complication rates between conventional versus robotic-assisted laparoscopy in the evaluation and management of early, advanced, and recurrent stage ovarian, fallopian tube, and primary peritoneal cancer. Int J Gynecol Cancer 2014;24:600-7.

24. Feuer GA, Lakhi N, Barker J, Salmieri S, Burrell M. Perioperative and clinical outcomes in the management of epithelial ovarian cancer using a robotic or abdominal approach. Gynecol Oncol 2013;131:520-4.

25. Ackroyd SA, Thomas S, Angel C, Moore R, Meacham PJ, DuBeshter B. Interval robotic cytoreduction following neoadjuvant chemotherapy in advanced ovarian cancer. 


\section{Obstetrics \& Gynecology Science}

Semiramis L. Carbajal-Mamani, et al. Robotic-assisted cytoreduction in ovarian cancer

J Robot Surg 2018;12:245-50.

26. Tozzi R, Gubbala K, Majd HS, Campanile RG. Interval laparoscopic en-bloc resection of the pelvis (L-EnBRP) in patients with stage IIIC-IV ovarian cancer: description of the technique and surgical outcomes. Gynecol Oncol 2016;142:477-83.

27. Favero G, Macerox N, Pfiffer T, Köhler C, da Costa Miranda $\mathrm{V}$, Estevez Diz MP, et al. Oncologic concerns regarding laparoscopic cytoreductive surgery in patients with advanced ovarian cancer submitted to neoadjuvant chemotherapy. Oncology 2015;89:159-66.

28. Corrado G, Mancini E, Cutillo G, Baiocco E, Vici P, Sergi $D$, et al. Laparoscopic debulking surgery in the management of advanced ovarian cancer after neoadjuvant chemotherapy. Int J Gynecol Cancer 2015;25:1253-7.

29. Cardenas-Goicoechea J, Wang Y, McGorray S, Saleem
MD, Carbajal Mamani SL, Pomputius AF, et al. Minimally invasive interval cytoreductive surgery in ovarian cancer: systematic review and meta-analysis. J Robot Surg 2019;13:23-33.

30. Brown J, Drury L, Crane EK, Anderson WE, Tait DL, Higgins $R V$, et al. When less is more: minimally invasive surgery compared with laparotomy for interval debulking after neoadjuvant chemotherapy in women with advanced ovarian cancer. J Minim Invasive Gynecol 2019;26:902-9.

31. Fagotti A, Gueli Alletti S, Corrado G, Cola E, Vizza E, Vieira $M$, et al. The INTERNATIONAL MISSION study: minimally invasive surgery in ovarian neoplasms after neoadjuvant chemotherapy. Int J Gynecol Cancer 2019;29:5-9. 